\title{
A new analytical approach to investigate human gait dynamics
}

\author{
Denisa Mihut ${ }^{1 *}$, and Nicolae Herisanu ${ }^{1,}$ \\ ${ }^{1}$ University Politehnica Timisoara, 300222 Bd. M. Viteazu, Timisoara, Romania
}

\begin{abstract}
In this paper we propose a new analytical approach to the study of human gait dynamics. A new and reliable method, namely the Optimal Auxiliary Functions Method (OAFM) is employed to obtain explicit and accurate analytical solutions. The capabilities of this new method are successfully tested in the study of human gait dynamics and an excellent agreement between analytical and numerical solutions is demonstrated. The accuracy of the analytical results is assured by the so-called convergence-control parameters, whose optimal values are rigorously identified in order to provide a fast convergence to the exact solution.
\end{abstract}

\section{Introduction}

The dynamics of human gait is a topic of interest for scientists concerned with biomechanics. It is known that human walking is actually a consequence of the commands given by the central nervous system transmitted through the intraspinal nervous system to the mechanical periphery of the body, which consists of bones and muscles receiving commands.

Many studies were recently developed on this subject using various methods of investigation. A comprehensive review of computational models applied in the study of human walking was presented by Ren et al. [1]. Perc [2] investigated the dynamics of human gait with simple nonlinear time series analysis methods. The direct collocation method was employed in [3] to investigate optimality principles for model-based prediction of human gait. Experimental investigations were also developed using different experimental techniques, such as those reported in [4], [5], [6].

Various types of non-linear oscillators can be used as Central Pattern Generators (CPG) to emphasize the trajectories of hip and knee in gait pattern. It was shown by Scafetta et al. [7] that a Van der Pol oscillator, which is in fact a prototypical nonlinear oscillator capable of producing stable oscillations, describes the quasiperiodic gait dynamics very well. This type of oscillator is widely used in many studies [8], [9].

In this work, taking into consideration the model proposed in [7], we develop a new analytical approach to the study of human gait dynamic problems by means of a new analytical method, namely the Optimal Auxiliary Functions Method (OAFM), in order to test its applicability in this kind of problems. The paper is focused on exploring a Van der Pol type non-linear oscillator used as Central Pattern Generator. The gait phenomenon is a

* Corresponding author: denisa mihut 01@yahoo.com 
rhythmic cycle, the movement of the hip while walking being oscillatory and it is known that the Central Pattern Generator (CPG) located in the nervous system is responsible for such oscillatory motions. An explicit analytical solution is expected for the model governed by the equation [7]

$$
\ddot{x}+\mu\left(x^{2}-p^{2}\right) \dot{x}+\left(2 \pi f_{j}\right)^{2} x=A \sin \left(2 \pi f_{0} t\right)
$$

with the initial conditions

$$
x(0)=B, \dot{x}(0)=0
$$

where $p$ is a parameter which controls the amplitude of the oscillations, the coefficient $\mu$ controls the degree of nonlinearity of the oscillator, $f_{j}$ is the virtual frequency of the oscillator during the $j$ th cycle, and $A$ and $f_{0}$ are the strength and frequency of the external driver, respectively.

\section{Basics of the Optimal Auxiliary Functions Method [10], [11]}

In order to briefly present the basics of the method employed in this study, namely the Optimal Auxiliary Functions Method (OAFM), we will take into consideration the most general form of a nonlinear differential equation

$$
L[u(x)]+g(x)+N[u(x)]=0
$$

with the initial conditions

$$
B\left(u(x), \frac{d u(x)}{d x}\right)=0
$$

It is well-known that an exact solution rarely happens to be identifiable for such an equation, so that in the frame of this method of solution, an approximate analytical solution will be proposed of the form

$$
\tilde{u}\left(x, C_{i}\right)=u_{0}(x)+u_{1}\left(x, C_{i}\right), \quad i=1,2, \ldots, s
$$

where $u_{0}(x)$ is the initial approximation and $u_{1}\left(x, C_{i}\right)$, which will be determined as follows.

After substituting Eq.(5) in (3) we obtain

$$
L\left(u_{0}(x)\right)+L\left(u_{1}\left(x, C_{i}\right)\right)+g(x)+N\left[u_{0}(x)+u_{1}\left(x, C_{i}\right)\right]=0
$$

At this stage, the initial approximation $u_{0}(x)$ is rigorously and easily obtained from the linear equation

$$
\begin{aligned}
& L\left[u_{0}(x)\right]+g(x)=0 \\
& B\left(u_{0}, \frac{d u_{0}}{d x}\right)=0
\end{aligned}
$$

while the first-order approximation is obtained from 


$$
\begin{aligned}
& L\left[u_{1}\left(x, C_{i}\right)\right]+N\left[u_{0}(x)+u_{1}\left(x, C_{i}\right)\right]=0 \\
& B\left(u_{1}, \frac{d u_{1}}{d x}\right)=0
\end{aligned}
$$

Having in view Eq.(8), the nonlinear term can be expanded in the form

$$
N\left[u_{0}(x)+u_{1}\left(x, C_{i}\right)\right]=N\left[u_{0}(x)\right]+\sum_{k=1}^{\infty} \frac{u_{1}^{k}}{k !} N^{(k)}\left[u_{0}(x)\right]
$$

In order to avoid expected difficulties appearing in solving Eq.(7), which could be strongly nonlinear, and also to accelerate the convergence of the first approximation $u_{1}$, instead of the last term from Eq.(8) we propose another expression so that Eq.(8) becomes

$$
\begin{aligned}
& L\left[u_{1}\left(x, C_{i}\right)\right]+A_{1}\left(u_{0}(x), u_{0}^{\prime}(x), C_{i}\right) N\left[u_{0}(x)\right]+A_{2}\left(u_{0}(x), u_{0}^{\prime}(x), C_{j}\right)=0 \\
& B\left(u_{1}(x), \frac{d u_{1}(x)}{d x}\right)=0
\end{aligned}
$$

in which $A_{1}$ and $A_{2}$ are auxiliary functions, characteristic for this method. These arbitrary functions depend on the initial approximation and also on several convergence-control parameters $C_{i}$ and $C_{j}, \mathrm{i}=1,2, \ldots, \mathrm{s}, \mathrm{j}=\mathrm{s}+1, \mathrm{~s}+2, \ldots, \mathrm{p}$. The optimal auxiliary functions $\mathrm{A}_{1}$ and $\mathrm{A}_{2}$ are not unique and, basically, they are of the same form like the initial approximation $u_{0}$. Therefore, if the initial approximation is an exponential function, then $A_{1}$ and $A_{2}$ are a sum of exponential functions. The same similitude applies when the initial approximation is a polynomial or a trigonometric function, in which case $A_{1}$ and $A_{2}$ are polynomial and trigonometric sums of functions, respectively. A particular case, which rarely happens for nonlinear differential equations, is the case when $N\left[u_{0}(x)\right]=0$, where obviously $u_{0}$ is an exact solution of the equation under study.

As a next step, the optimal values of the initially unknown convergence-control parameters should be identified using various rigorous methods, such as the Galerkin method, the least square method, the Ritz method, the collocation method and so on. Knowing these optimal values of $C_{i}$ and $C_{j}$, the approximate analytical solution is explicitly obtained, so that this procedure proves to be a powerful tool for solving nonlinear and even strongly nonlinear problems.

\section{Application of the OAFM}

Equations should be centred and should be numbered with the number on the right-hand side.

$$
\begin{gathered}
\tau=\Omega t, \quad B u(\tau)=x(t) \\
u^{\prime \prime}+\frac{\mu}{\Omega}\left(B^{2} u^{2}-p^{2}\right) u^{\prime}+\frac{4 \pi^{2} f_{j}^{2}}{\Omega^{2}} u=\frac{A}{\Omega^{2} B} \sin \frac{2 \pi f_{0}}{\Omega} \tau \\
u(0)=1, \quad u^{\prime}(0)=0
\end{gathered}
$$

To identify a convenient linear and nonlinear term, Eq. (12) may be re-written as 


$$
u^{\prime \prime}+u+\frac{\mu}{\Omega}\left(B^{2} u^{2}-p^{2}\right) u^{\prime}+\left(\frac{4 \pi^{2} f_{j}^{2}}{\Omega^{2}}-1\right) u-\frac{A}{\Omega^{2} B} \sin \frac{2 \pi f_{0}}{\Omega} \tau=0
$$

so that one can be identified the linear and nonlinear terms as

$$
\begin{aligned}
& L(u)=u^{\prime \prime}+u \\
& N(u)=\frac{\mu}{\Omega}\left(B^{2} u^{2}-p^{2}\right) u^{\prime}+\left(\frac{4 \pi^{2} f_{j}^{2}}{\Omega^{2}}-1\right) u-\frac{A}{\Omega^{2} B} \sin \frac{2 \pi f_{0}}{\Omega} \tau
\end{aligned}
$$

The initial approximation $\mathrm{u}_{0}(\tau)$ may be identified now from the linear equation

$$
L\left(u_{0}\right)=0, u_{0}(0)=1, \quad u_{0}^{\prime}(0)=0
$$

which becomes

$$
u_{0}^{\prime \prime}+u_{0}=0
$$

and has the solution

$$
u_{0}(\tau)=\cos \tau
$$

Consequently, taking into account this result, the nonlinear term becomes

$$
\begin{aligned}
& N\left(u_{0}\right)=-\frac{\mu}{\Omega^{2}}\left[\frac{B^{2}}{2}(1+\cos 2 \tau)-p^{2}\right] \sin \tau+\left(\frac{4 \pi^{2} f_{j}^{2}}{\Omega^{2}}-1\right) \cos \tau-\frac{A}{\Omega^{2} B} \sin \frac{2 \pi f_{0}}{\Omega} \tau= \\
& =-\frac{\mu B^{2}}{4 \Omega^{2}}(\sin \tau+\sin 3 \tau)+\frac{\mu p^{2}}{\Omega^{2}} \sin \tau+\left(\frac{4 \pi^{2} f_{j}^{2}}{\Omega^{2}}-1\right) \cos \tau-\frac{A}{\Omega^{2} B} \sin \frac{2 \pi f_{0}}{\Omega} \tau= \\
& =\frac{4 \mu p^{2}-\mu B^{2}}{4 \Omega^{2}} \sin \tau+\left(\frac{4 \pi^{2} f_{j}^{2}}{\Omega^{2}}-1\right) \cos \tau-\frac{\mu B^{2}}{\Omega^{2}} \sin 3 \tau-\frac{A}{\Omega^{2} B} \sin \frac{2 \pi f_{0}}{\Omega} \tau
\end{aligned}
$$

or by denoting

$$
M_{1}=\frac{4 \mu p^{2}-\mu B^{2}}{4 \Omega^{2}}, \quad N_{1}=\frac{4 \pi^{2} f_{j}^{2}}{\Omega^{2}}-1, \quad M_{3}=-\frac{\mu B^{2}}{4 \Omega^{2}}, \quad Q=-\frac{A}{\Omega^{2} B}
$$

the Eq.(19) becomes

$$
N\left(u_{0}\right)=M_{1} \sin \tau+N_{1} \cos \tau+M_{3} \sin 3 \tau+Q \sin \frac{2 \pi f_{0}}{\Omega} \tau
$$

Taking into account Eq.(10) and the described procedure, we propose the expression

$$
\begin{aligned}
& L u_{1}=2 C_{1} \sin 2 \tau\left(M_{1} \sin \tau+N_{1} \cos \tau+M_{3} \sin 3 \tau\right)+C_{2} \sin \tau+ \\
& +C_{3} \cos \tau+C_{4} \sin 3 \tau+C_{5} Q \sin \frac{2 \pi f_{0}}{\Omega} \tau
\end{aligned}
$$

and after simple manipulations we obtain 


$$
\begin{aligned}
& u_{1}^{\prime \prime}+u_{1}=M_{1} C_{1}(\cos \tau-\cos 3 \tau)+N_{1} C_{1}(\sin 3 \tau+\sin \tau)+M_{3} C_{1}(\cos \tau-\cos 5 \tau)+ \\
& +C_{2} \sin \tau+C_{3} \cos \tau+C_{4} \sin 3 \tau+Q C_{5} \sin \frac{2 \pi f_{0}}{\Omega} \tau= \\
& =\left(M_{1} C_{1}+M_{3} C_{1}+C_{3}\right) \cos \tau+\left(N_{1} C_{1}+C_{2}\right) \sin \tau+\left(N_{1} C_{1}+C_{4}\right) \sin 3 \tau-M_{1} C_{1} \cos 3 \tau- \\
& -M_{3} C_{1} \cos 5 \tau+Q C_{5} \sin \frac{2 \pi f_{0}}{\Omega} \tau
\end{aligned}
$$

In order to avoid the presence of secular terms, it is needed that:

$$
\frac{2 \mu p^{2}-\mu B^{2}}{2 \Omega^{2}} C_{1}+C_{3}=0,\left(\frac{4 \pi^{2} f_{j}^{2}}{\Omega^{2}}-1\right) C_{1}+C_{2}=0
$$

Finally, the differential equation corresponding to the first order approximate solution $\mathrm{u}_{1}$ can be written as

$$
\begin{aligned}
& u_{1}^{\prime \prime}+u_{1}=\left(N_{1} C_{1}+C_{4}\right) \sin 3 \tau-M_{1} C_{1} \cos 3 \tau-M_{3} C_{1} \cos 5 \tau+Q C_{5} \sin \frac{2 \pi f_{0}}{\Omega} \tau \\
& u_{1}^{\prime}(0)=u_{1}(0)=0
\end{aligned}
$$

which has the solution

$$
\begin{aligned}
& u_{1}(\tau)=\frac{N_{1} C_{1}+C_{4}}{8}(3 \sin \tau-\sin 3 \tau)-\frac{M_{1} C_{1}}{8}(\cos \tau-\cos 3 \tau)- \\
& -\frac{M_{3} C_{1}}{24}(\cos \tau-\cos 5 \tau)+\frac{Q C_{5} \Omega^{2}}{\Omega^{2}-4 \pi^{2} f_{0}^{2}}\left(\sin \frac{2 \pi f_{0}}{\Omega} \tau-\frac{2 \pi f_{0}}{\Omega} \sin \tau\right)
\end{aligned}
$$

and according to the described procedure, the first-order approximate solution will be

$$
u_{O A F M}\left(\tau, C_{i}\right)=u_{0}(\tau)+u_{1}\left(\tau, C_{i}\right), \quad i=1,2, \ldots, 5
$$

where the optimal values of the convergence-control parameters remain to be identified for any specific values of the physical parameters.

In order to develop a numerical example with the help of Mathematica software, with the aim to prove the accuracy of the obtained solution, the following set of physical parameters is considered: $\mu=1.2, \mathrm{p}=0.9, \mathrm{f}_{\mathrm{j}}=1.1$. At this stage, using the procedures described in [10], [12], the optimal values of the initially unknown parameters are:

$$
\begin{aligned}
& \mathrm{C}_{1}=1.449398869634, C_{2}=0.080506333361, C_{3}=0.021836308426, \\
& C_{4}=0.208647756498, C_{5}=3.225952886784, \Omega=6.904597698444
\end{aligned}
$$

and therefore, the final expression of the analytical approximate solution will be

$$
\begin{aligned}
& \tilde{x}(t)=1.70119 \cos [\Omega \mathrm{t}]+0.0924378 \sin [\Omega \mathrm{t}]+0.000678359 \cos [3 \Omega \mathrm{t}]- \\
& -0.0449541 \sin [3 \Omega \mathrm{t}]-0.0018671 \cos [5 \Omega \mathrm{t}]+0.0133201 \sin \left[2 \pi f_{0} \mathrm{t}\right]
\end{aligned}
$$

In fig. 1 is presented a comparison between the proposed approximate analytical solution obtained through OAFM and numerical integration results obtained by means of a fourth-order Runge-Kutta method for the considered case. 


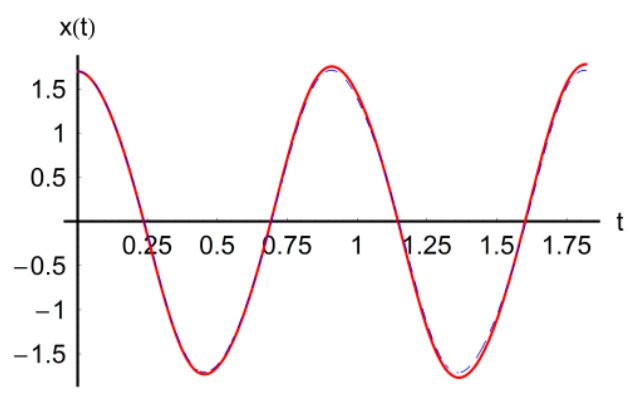

Fig. 1. Comparison between analytical and numerical integration results: fourth-order RK results analytical results;

One can be seen from fig. 1 the excellent accuracy of the approximate analytical results, which almost overlap the numerical integration results obtained by means of a fourth-order Runge-Kutta method, which proves the efficiency of the proposed method. The plot is represented only for two periods of the motion, the elongation $\mathrm{x}(\mathrm{t})$ having the same behaviour in time $t$ also for large time.

\section{Conclusions}

An explicit analytical solution is obtained for the nonlinear dynamical system describing the human gait, which is very useful since it allows a deeper insight into the dynamics of the locomotor system. A new effective analytical approach is proposed, namely the Optimal Auxiliary Functions Method, whose capabilities were successfully tested. The accuracy of the obtained solution is proved through comparisons with numerical integration results, which demonstrate the applicability of the proposed approach.

\section{References}

1. L. Ren, D. Howard, L. Kenney, J Bionic Eng. 3, 127 (2006)

2. M. Perc, Eur. J. Phys. 26, 525 (2005)

3. M. Ackermann, A.J. Bogert, J. Biomech. 43, 1055 (2010)

4. D.I. Stoia, C. Vigaru, L. Rusu, Springer Proc. Phys. 198, 201 (2018)

5. C. Kirtley, Clinical Gait Analysis, Theory and Practice (Elsevier, London, 2006)

6. R.E. Mayagoitia, A.V. Nene, P.H. Veltink, J. Biomech. 35, 537 (2002)

7. N. Scafetta, D. Marchi, B.J. West, Chaos, 026108 (2009)

8. J. Bay, H. Hemami, IEEE Trans. Biomed. Eng. 4, 297 (1987)

9. L. Cveticanin, Appl. Mech. Mater. 430, 3 (2013)

10. N. Herisanu, V. Marinca, G. Madescu, F. Dragan, Energies. 12, 915, (2019)

11. N. Herisanu, V. Marinca, Springer Proc. Phys. 198, 41, (2018)

12. V. Marinca, N. Herisanu, Int. J. Heat and Mass Transf. 79 146, (2014) 